\title{
Stancu type $q$-Bernstein operators with shifted knots
}

\author{
M. Mursaleen ${ }^{1,2,3^{*}}$ (D) Mohd Qasim ${ }^{4}$, Asif Khan ${ }^{3}$ and Zaheer Abbas ${ }^{4}$
}

\author{
"Correspondence: \\ mursaleenm@gmail.com \\ 'Department of Medical Research, \\ China Medical University Hospital, \\ China Medical University (Taiwan), \\ Taichung, Taiwan \\ ${ }^{2}$ Department of Computer Science \\ and Information Engineering, Asia \\ University, Taichung, Taiwan \\ Full list of author information is \\ available at the end of the article
}

\begin{abstract}
In the present paper, Stancu type generalizations of the $q$-analog of Lupaş Bernstein operators with shifted knots are introduced. Some approximation results and rate of convergence for these operators are investigated. A Voronovskaja type theorem and local approximation results for the mentioned operators are studied. The extra parameters $\gamma, \delta, q, a$ and $b$ provide more flexibility for approximation.
\end{abstract}

MSC: 41A10; 41A25; 41A36

Keywords: Lupaş q-Bernstein-Stancu shifted operators; Rate of convergence; Modulus of continuity; Voronovskaja type theorem; $K$-functional; Local approximation

\section{Introduction}

Approximation theory basically deals with approximation of functions by simpler functions or more easily calculated functions. Broadly it is divided into theoretical and constructive approximation. In 1912 Bernstein [5] was the first to construct a sequence of positive linear operators as follows:

$$
B_{m}(f ; u)=\sum_{k=0}^{m}\left(\begin{array}{l}
m \\
k
\end{array}\right) u^{k}(1-u)^{m-k} f\left(\frac{k}{m}\right),
$$

where $u \in[0,1], f$ is bounded on $[0,1]$.

A constructive proof of the well-known Weierstrass approximation theorem using a probabilistic approach was provided. Here $C[0,1]$ denotes the set of all continuous functions on $[0,1]$ which is equipped with the sup-norm $\|\cdot\|$. He showed that if $f \in C[0,1]$, then $B_{m}(f ; u)$ converges to $f(u)$ uniformly on $[0,1]$. One can find a detailed monograph about the Bernstein polynomials in $[12,13]$.

Before proceeding, let us recall some basic definitions and notations of quantum calculus [9]. For any fixed real number $q>0$ satisfying the conditions $0<q \leq 1$, the $q$-integer $[k]_{q}$, for $k \in \mathbb{N}$ is defined as

$$
[k]_{q}:= \begin{cases}\frac{\left(1-q^{k}\right)}{(1-q)}, & q \neq 1, \\ k, & q=1\end{cases}
$$

(c) The Author(s) 2020. This article is licensed under a Creative Commons Attribution 4.0 International License, which permits use, sharing, adaptation, distribution and reproduction in any medium or format, as long as you give appropriate credit to the original author(s) and the source, provide a link to the Creative Commons licence, and indicate if changes were made. The images or other third party material in this article are included in the article's Creative Commons licence, unless indicated otherwise in a credit line to the material. If material is not included in the article's Creative Commons licence and your intended use is not permitted by statutory regulation or exceeds the permitted use, you will need to obtain permission directly from the copyright holder. To view a copy of this licence, visit http://creativecommons.org/licenses/by/4.0/. 
and the $q$-factorial by

$$
[k]_{q} !:= \begin{cases}{[k]_{q}[k-1]_{q} \cdots[1]_{q},} & k \geq 1, \\ 1, & k=0 .\end{cases}
$$

The $q$-binomial expansion is

$$
(u+y)_{q}^{m}:=(u+y)(u+q y)\left(u+q^{2} y\right) \cdots\left(u+q^{m-1} y\right),
$$

and the $q$-binomial coefficients are as follows:

$$
\left[\begin{array}{l}
m \\
k
\end{array}\right]_{q}:=\frac{[m]_{q} !}{[k]_{q} ![m-k]_{q} !} .
$$

From the above

$$
(u)_{q}^{m}=u^{m}
$$

and

$$
(y)_{q}^{m}:=(y)(q y)\left(q^{2} y\right) \cdots\left(q^{m-1} y\right)=q^{\frac{m(m-1)}{2}} y^{m} .
$$

Gauss-formula is defined as

$$
(u+y)_{q}^{m}=\sum_{j=0}^{m}\left[\begin{array}{c}
m \\
k
\end{array}\right]_{q} q^{j(j-1) / 2} y^{j} u^{m-j} .
$$

After development of $q$-calculus, Lupaş [14] in 1987 introduced the $q$-Lupaş operator (rational) as follows:

$$
L_{m, q}(f ; u)=\sum_{k=0}^{m} \frac{f\left(\frac{[k]_{q}}{[m]_{q}}\right)\left(\begin{array}{c}
m \\
k
\end{array}\right)_{q} q^{\frac{k(k-1)}{2}} u^{k}(1-u)^{m-k}}{\prod_{j=1}^{m}\left\{(1-u)+q^{j-1} u\right\}},
$$

and studied its approximation properties.

Similarly, Phillips [23] in 1996 constructed another $q$-analog of Bernstein operators (polynomials) as follows:

$$
B_{m, q}(f ; u)=\sum_{k=0}^{m}\left[\begin{array}{c}
m \\
k
\end{array}\right]_{q} u^{k} \prod_{s=0}^{m-k-1}\left(1-q^{s} x\right) f\left(\frac{[k]_{q}}{[m]_{q}}\right), \quad x \in[0,1],
$$

where $B_{m, q}: C[0,1] \rightarrow C[0,1]$ defined for any $m \in \mathbb{N}$ and any function $f \in C[0,1]$.

Bases of these operators have been used in computer aided geometric design (CAGD) to study curves and surfaces. From then onward it became an active area of research in approximation theory as well as CAGD. In the recent past, $q$-analogs of various operators were investigated by several researchers (see $[6,15,19,22,24])$. Also see $[1,2,4,10,11,16-$ $18,21,25]$ for other modifications. 
In 1968 Stancu [26] showed that the Bernstein-Stancu polynomials

$$
\left(P_{m}^{(\gamma, \delta)} f\right)(u)=\sum_{k=0}^{m}\left(\begin{array}{l}
m \\
k
\end{array}\right) u^{k}(1-u)^{m-k} f\left(\frac{k+\gamma}{m+\delta}\right)
$$

converge to continuous function $f(u)$ uniformly in $[0,1]$ for each real $\gamma, \delta$ such that $0 \leq$ $\gamma \leq \delta$.

In 2010, a new construction of Bernstein-Stancu type polynomials with shifted knots was introduced by Gadjiev and Gorhanalizadeh [8]:

$$
\begin{aligned}
S_{m, \gamma, \delta}(f ; u)= & \left(\frac{m+\delta_{2}}{m}\right)^{m} \sum_{k=0}^{m}\left(\begin{array}{c}
m \\
k
\end{array}\right)\left(u-\frac{\gamma_{2}}{m+\delta_{2}}\right)^{k}\left(\frac{m+\gamma_{2}}{m+\delta_{2}}-u\right)^{m-k} \\
& \times f\left(\frac{k+\gamma_{1}}{m+\delta_{1}}\right),
\end{aligned}
$$

where $\frac{\gamma_{2}}{n+\delta_{2}} \leq u \leq \frac{n+\gamma_{2}}{n+\delta_{2}}$ and $\gamma_{k}, \delta_{k}(k=1,2)$ are positive real numbers provided $0 \leq \gamma_{1} \leq$ $\gamma_{2} \leq \delta_{1} \leq \delta_{2}$. It is clear that, for $\gamma_{2}=\delta_{2}=0$, the polynomials (1.6) turn into the BernsteinStancu polynomials (1.5) and if $\gamma_{1}=\gamma_{2}=\delta_{1}=\delta_{2}=0$ then these polynomials turn into the classical Bernstein polynomials.

Khalid et al. studied Bezier curves and surfaces using basis of shifted Bernstein polynomial in [11]. Recently, Mursaleen et al. [20] introduced and studied Lupaş Bernstein shifted operators based on $q$-integers as follows:

$$
\begin{aligned}
S_{m, q}^{(\gamma, \delta)}(f ; u)= & \frac{1}{\left(\frac{[m]_{q}}{[m]_{q}+\delta}\right)_{q}^{m}} \sum_{j=0}^{m}\left[\begin{array}{c}
m \\
j
\end{array}\right]_{q} q^{\frac{j(j-1)}{2}}\left(\frac{[m]_{q}+\gamma}{[m]_{q}+\delta}-u\right)^{m-j} \\
& \times\left(u-\frac{a}{[m]_{q}+b}\right)^{j} f\left(\frac{[j]_{q}}{[m]_{q}}\right)
\end{aligned}
$$

or

$$
S_{m, q}^{(\gamma, \delta)}(f ; u)=\frac{1}{\left(\frac{[m]_{q}}{[m]_{q}+\delta}\right)_{q}^{m}} \sum_{j=0}^{m}\left[\begin{array}{c}
m]_{q} \\
j
\end{array}\right]_{q}^{[m]_{q}+\gamma} \frac{[m]_{q}+\delta}{[m-j}\left(u-\frac{a}{[m]_{q}+\delta}\right)^{j} f\left(\frac{[j]_{q}}{[m]_{q}}\right)
$$

where $\frac{\gamma}{[m]_{q}+\delta} \leq u \leq \frac{[m]_{q}+\gamma}{[m]_{q}+\delta}$ and $\gamma, \delta$ are positive real numbers provided $0 \leq \gamma \leq \delta$. In case $\gamma=\delta=0$, the above operators (1.7) reduce to Lupaş $q$-Bernstein operators [14].

Motivated by the above work, in the next section we present a Stancu type modification of Lupaş $q$-Bernstein shifted operators and will study its approximation properties.

\section{Construction of Lupaş 9 -Bernstein-Stancu shifted operators}

Let $\gamma, \delta \in \mathbb{N}_{0}$ (the set of all non-negative integers) be such that $0 \leq \gamma \leq \delta$ and $0 \leq a \leq b$, then we have

$$
\begin{aligned}
S_{m, q}^{(\gamma, \delta, a, b)}(f ; u)= & \frac{1}{\left(\frac{[m]_{q}}{[m]_{q}+\delta}\right)_{q}^{m}} \sum_{k=0}^{m}\left[\begin{array}{c}
m \\
k
\end{array}\right]_{q} q^{\frac{k(k-1)}{2}}\left(u-\frac{\gamma}{[m]_{q}+\delta}\right)^{k} \\
& \times\left(\frac{[m]_{q}+\gamma}{[m]_{q}+\delta}-u\right)^{m-k} f\left(\frac{[k]_{q}+a}{[m]_{q}+b}\right)
\end{aligned}
$$


or

$$
\begin{aligned}
& S_{m, q}^{(\gamma, \delta, a, b)}(f ; u) \\
& \quad=\frac{1}{\left(\frac{[m]_{q}}{[m]_{q}+\delta}\right)_{q}^{m}} \sum_{k=0}^{m}\left[\begin{array}{c}
m \\
k
\end{array}\right]_{q}\left(u-\frac{\gamma}{[m]_{q}+\delta}\right)_{q}^{k}\left(\frac{[m]_{q}+\gamma}{[m]_{q}+\delta}-u\right)^{m-k} f\left(\frac{[k]_{q}+a}{[m]_{q}+b}\right),
\end{aligned}
$$

where $\frac{\gamma}{[m]_{q}+\delta} \leq u \leq \frac{[m]_{q}+\gamma}{[m]_{q}+\delta}$ and $\gamma, \delta$ are positive real numbers provided $0 \leq \gamma \leq \delta$. In the case $a=b=0$, the above operators (2.1) reduces to (1.7).

\section{Definitions and auxiliary results}

Lemma 3.1 Let $S_{m, q}^{(\gamma, \delta, a, b)}(f ; u)$ be given by (2.1). Then the following properties hold:

(1) $S_{m, q}^{(\gamma, \delta, a, b)}(1 ; u)=1$,

(2) $S_{m, q}^{(\gamma, \delta, a, b)}(t ; u)=\frac{[m]_{q}+\delta}{[m]_{q}+b}\left(u-\frac{\gamma}{[m]_{q}+\delta}\right)+\frac{a}{[m]_{q}+b}$,

(3) $S_{m, q}^{(\gamma, \delta, a, b)}\left(t^{2} ; u\right)=\left(\frac{q^{2}[m-1]_{q}}{[m]_{q}+b}\right)\left(\frac{[m]_{q}+\delta}{[m]_{q}+b}\right) \frac{\left(u-\frac{\gamma}{[m]_{q}+\delta}\right)^{2}}{\left\{\frac{[m]_{q}+\gamma}{[m]_{q}+\delta}-u+q\left(u-\frac{\gamma}{[m]_{q}+\delta}\right)\right\}}+\left(\frac{[m]_{q}+\delta}{\left([m]_{q}+b\right)^{2}}\right)\left(u-\frac{\gamma}{[m]_{q}+\delta}\right)+$ $\frac{a}{\left([m]_{q}+b\right)^{2}}$

(4) $S_{m, q}^{(\gamma, \delta, a, b)}\left(t^{3} ; u\right)=\left(\frac{[m]_{q}+\delta}{[m]_{q}+b}\right) \frac{q^{6}[m-1]_{q}[m-2]_{q}\left(u-\frac{\gamma}{[m]_{q}+\delta}\right)^{3}}{\left([m]_{q}+b\right)^{2}\left\{\frac{[m]_{q}+\gamma}{[m]_{q}+\delta}-u+q\left(u-\frac{\gamma}{[m] q+\delta}\right)\right\}\left\{\frac{[m]_{q}+\gamma}{[m]_{q}+\delta}-u+q^{2}\left(u-\frac{\gamma}{[m]_{q}+\delta}\right)\right\}}+(2+3 a+$ q) $\left(\frac{q^{2}[m-1]_{q}}{\left([m]_{q}+b\right)^{2}}\right)\left(\frac{[m]_{q}+\delta}{[m]_{q}+b}\right) \frac{\left(u-\frac{\gamma}{[m]_{q}+\delta}\right)^{2}}{\left\{\frac{[m]_{q}+\gamma}{[m]_{q}+\delta}-u+q\left(u-\frac{\gamma}{[m]_{q}+\delta}\right)\right\}}+\left(1+3 a^{2}\right)\left(\frac{[m]_{q}+\delta}{\left([m]_{q}+b\right)^{3}}\right)\left(u-\frac{\gamma}{[m]_{q}+\delta}\right)+\frac{a^{3}}{\left([m]_{q}+b\right)^{3}}+$ $\frac{3 a^{2}\left([m]_{q}+\delta\right)}{\left([m]_{q}+b\right)^{3}}$,

(5) $S_{m, q}^{(\gamma, \delta, a, b)}\left(t^{4} ; u\right)=\frac{q^{12}[m-1]_{q}[m-2]_{q}[m-3]_{q}}{\left([m]_{q}+b\right)^{3}}\left(\frac{[m]_{q}+\delta}{[m]_{q}+b}\right) \times \frac{1}{\left\{\frac{[m]_{q}+\gamma}{[m]_{q}+\delta}-u+q\left(u-\frac{\gamma}{[m]_{q}+\delta}\right)\right\}} \times$

$$
\begin{aligned}
& \frac{\left(u-\frac{\gamma}{[m]_{q}+\delta}\right)^{4}}{\left\{\frac{[m]_{q}+\gamma}{[m]_{q}+\delta}-u+q^{2}\left(u-\frac{\gamma}{[m]_{q}+\delta}\right)\right\}\left(\frac{m]_{q}+\gamma}{[m]_{q}+\delta}-u+q^{3}\left(u-\frac{\gamma}{[m]_{q}+\delta}\right)\right.}+\frac{[m-1]_{q}[m-2]_{q}}{\left([m]_{q}+b\right)^{3}}\left(\frac{[m]_{q}+\delta}{[m]_{q}+b}\right) \times \\
& \frac{\left(q^{8}+2 q^{7}+3 q^{6}+4 a q^{2}\right)\left(u-\frac{\gamma}{[m]_{q}+\delta}\right)^{3}}{\left\{\frac{[m]_{q}+\gamma}{[m]_{q}+\delta}-u+q\left(u-\frac{\gamma}{[m]_{q}+\delta}\right)\right\}\left\{\frac{[m]_{q}+\gamma}{[m]_{q}+\delta}-u+q^{2}\left(u-\frac{\gamma}{[m]_{q}+\delta}\right)\right\}}+\left(q^{4}+3 q^{3}+3 q^{2}+6 a^{2} q^{2}\right) \frac{[m-1]_{q}}{\left([m]_{q}+b\right)^{3}}\left(\frac{[m]_{q}+\delta}{[m]_{q}+b}\right) \times \\
& \frac{\left(u-\frac{\gamma}{[m]_{q}+\delta}\right)^{2}}{\left\{\frac{(m]_{q}+\gamma}{[m]_{q}+\delta}-u+q\left(u-\frac{\gamma}{[m]_{q}+\delta}\right)\right.}+\left(1+4 a^{3}\right)\left(\frac{[m]_{q}+\delta}{\left([m]_{q}+b\right)^{4}}\right)\left(u-\frac{\gamma}{[m]_{q}+\delta}\right)+\frac{a^{4}}{\left([m]_{q}+b\right)^{4}}+\frac{6 a^{2}\left([m]_{q}+\delta\right)}{\left([m]_{q}+b\right)^{4}} .
\end{aligned}
$$

Proof (1) By using definition of $q$-binomial coefficients and Gauss-formula, we have

$$
\begin{aligned}
& S_{m, q}^{(\gamma, \delta, a, b)}(1 ; u) \\
& \quad=\frac{1}{\left(\frac{[m]_{q}}{[m]_{q}+\delta}\right)_{q}^{m}} \sum_{k=0}^{m}\left[\begin{array}{c}
m \\
k
\end{array}\right]_{q} q^{\frac{k(k-1)}{2}}\left(u-\frac{\gamma}{[m]_{q}+\delta}\right)^{k}\left(\frac{[m]_{q}+\gamma}{[m]_{q}+\delta}-u\right)^{m-k}=1 .
\end{aligned}
$$

(2) For $f(t)=t$, we have

$$
\begin{aligned}
& S_{m, q}^{(\gamma, \delta, a, b)}(t ; u) \\
& =\frac{1}{\left(\frac{[m]_{q}}{[m]_{q}+\delta}\right)_{q}^{m}} \sum_{k=0}^{m}\left[\begin{array}{c}
m \\
k
\end{array}\right]_{q} q^{\frac{k(k-1)}{2}}\left(u-\frac{\gamma}{[m]_{q}+\delta}\right)^{k}\left(\frac{[m]_{q}+\gamma}{[m]_{q}+\delta}-u\right)^{m-k}\left(\frac{[k]_{q}+a}{[m]_{q}+b}\right) \\
& =\frac{1}{\left([m]_{q}+b\right)\left(\frac{[m]_{q}}{[m]_{q}+\delta}\right)_{q}^{m}} \sum_{k=0}^{m-1}\left[\begin{array}{c}
m-1 \\
k
\end{array}\right]_{q} q^{\frac{k(k+1)}{2}}\left(u-\frac{\gamma}{[m]_{q}+\delta}\right)^{k+1}\left(\frac{[m]_{q}+\gamma}{[m]_{q}+\delta}-u\right)^{m-k-1}
\end{aligned}
$$


Mursaleen et al. Journal of Inequalities and Applications

(2020)

2020:28

Page 5 of 14

$$
\begin{aligned}
& +\frac{a}{\left([m]_{q}+b\right)\left(\frac{[m]_{q}}{[m]_{q}+\delta}\right)_{q}^{m}} \sum_{k=0}^{m}\left[\begin{array}{c}
m \\
k
\end{array}\right]_{q} q^{\frac{k(k-1)}{2}}\left(u-\frac{\gamma}{[m]_{q}+\delta}\right)^{k}\left(\frac{[m]_{q}+\gamma}{[m]_{q}+\delta}-u\right)^{m-k} \\
= & \frac{1}{\left(\frac{[m]_{q}}{[m]_{q}+\delta}\right)_{q}^{m}} \sum_{k=0}^{m-1}\left[\begin{array}{c}
m-1 \\
k
\end{array}\right]_{q} q^{\frac{k(k-1)}{2}}\left(\frac{q Y}{u}\right)^{k} u^{m-1} Y+\frac{a}{[m]_{q}+b} \\
= & \left(\frac{[m]_{q}+\delta}{[m]_{q}+b}\right) Y+\frac{a}{[m]_{q}+b}\left(\text { where } u=\frac{[m]_{q}+\gamma}{[m]_{q}+\delta}-u, Y=u-\frac{\gamma}{[m]_{q}+\delta}\right) \\
= & \left(\frac{[m]_{q}+\delta}{[m]_{q}+b}\right)\left(u-\frac{\gamma}{[m]_{q}+\delta}\right)+\frac{a}{[m]_{q}+b} .
\end{aligned}
$$

(3) For $f(t)=t^{2}$, we have

$$
\begin{aligned}
& S_{m, q}^{(\gamma, \delta, a, b)}\left(t^{2} ; u\right) \\
& =\frac{1}{\left(\frac{[m]_{q}}{[m]_{q}+\delta}\right)_{q}^{m}} \sum_{k=0}^{m}\left[\begin{array}{c}
m \\
k
\end{array}\right]_{q} q^{\frac{k(k-1)}{2}}\left(u-\frac{\gamma}{[m]_{q}+\delta}\right)^{k}\left(\frac{[m]_{q}+\gamma}{[m]_{q}+\delta}-u\right)^{m-k}\left(\frac{[k]_{q}+a}{[m]_{q}+b}\right)^{2} \\
& =\frac{1}{\left([m]_{q}+b\right)^{2}\left(\frac{[m]_{q}}{[m]_{q}+\delta}\right)_{q}^{m}} \sum_{k=0}^{m}\left[\begin{array}{c}
m \\
k
\end{array}\right]_{q} q^{\frac{k(k-1)}{2}}\left(u-\frac{\gamma}{[m]_{q}+\delta}\right)^{k} \\
& \times\left(\frac{[m]_{q}+\gamma}{[m]_{q}+\delta}-u\right)^{m-k}\left([k]_{q}+a\right)^{2} \\
& =\frac{1}{\left([m]_{q}+b\right)^{2}\left(\frac{[m]_{q}}{[m]_{q}+\delta}\right)_{q}^{m}} \sum_{k=0}^{m}\left[\begin{array}{c}
m \\
k
\end{array}\right]_{q} q^{\frac{k(k-1)}{2}}\left(u-\frac{\gamma}{[m]_{q}+\delta}\right)^{k}\left(\frac{[m]_{q}+\gamma}{[m]_{q}+\delta}-u\right)^{m-k}[k]_{q}{ }^{2} \\
& +\frac{a^{2}}{\left([m]_{q}+b\right)^{2}\left(\frac{[m]_{q}}{[m]_{q}+\delta}\right)_{q}^{m}} \sum_{k=0}^{m}\left[\begin{array}{c}
m \\
k
\end{array}\right]_{q} q^{\frac{k(k-1)}{2}}\left(u-\frac{\gamma}{[m]_{q}+\delta}\right)^{k}\left(\frac{[m]_{q}+\gamma}{[m]_{q}+\delta}-u\right)^{m-k} \\
& +\frac{2 a}{\left([m]_{q}+b\right)^{2}\left(\frac{[m]_{q}}{[m]_{q}+\delta}\right)_{q}^{m}} \sum_{k=0}^{m}\left[\begin{array}{c}
m \\
k
\end{array}\right]_{q} q^{\frac{k(k-1)}{2}}\left(u-\frac{\gamma}{[m]_{q}+\delta}\right)^{k} \\
& \times\left(\frac{[m]_{q}+\gamma}{[m]_{q}+\delta}-u\right)^{m-k}[k]_{q} \\
& =A+B+C \text { (say), }
\end{aligned}
$$

after calculating the values of $A, B$ and $C$ we get

$$
\begin{aligned}
& S_{m, q}^{(\gamma, \delta, a, b)}\left(t^{2} ; u\right) \\
& \quad=\left(\frac{[m]_{q}+\delta}{\left.([m]+b)_{q}\right)^{2}}\right)\left(u-\frac{\gamma}{[m]_{q}+\delta}\right) \\
& \quad+\left(\frac{q^{2}[m-1]_{q}}{[m]_{q}+b}\right)\left(\frac{[m]_{q}+\delta}{[m]_{q}+b}\right) \frac{\left(u-\frac{\gamma}{[m]_{q}+\delta}\right)^{2}}{\left\{\frac{[m]_{q}+\gamma}{[m]_{q}+\delta}-u+q\left(u-\frac{\gamma}{[m]_{q}+\delta}\right)\right\}}+\frac{a}{\left.([m]+b)_{q}\right)^{2}},
\end{aligned}
$$

which is the required result. 
(4) For $f(t)=t^{3}$, we have

$$
\begin{aligned}
& S_{m, q}^{(\gamma, \delta, a, b)}\left(t^{3} ; u\right) \\
& =\frac{1}{\left(\frac{[m]_{q}}{[m]_{q}+\delta}\right)_{q}^{m}} \sum_{k=0}^{m}\left[\begin{array}{c}
m \\
k
\end{array}\right]_{q} q^{\frac{k(k-1)}{2}}\left(u-\frac{\gamma}{[m]_{q}+\delta}\right)^{k}\left(\frac{[m]_{q}+\gamma}{[m]_{q}+\delta}-u\right)^{m-k}\left(\frac{[k]_{q}+a}{[m]_{q}+b}\right)^{3} \\
& =\frac{1}{\left.([m]+b)_{q}\right)^{3}\left(\frac{[m]_{q}}{[m]_{q}+\delta}\right)_{q}^{m}} \sum_{k=0}^{m}\left[\begin{array}{c}
m \\
k
\end{array}\right]_{q} q^{\frac{k(k-1)}{2}}\left(u-\frac{\gamma}{[m]_{q}+\delta}\right)^{k} \\
& \times\left(\frac{[m]_{q}+\gamma}{[m]_{q}+\delta}-u\right)^{m-k}\left([k]_{q}+a\right)^{3} \\
& =\frac{1}{\left.([m]+b)_{q}\right)^{3}\left(\frac{[m]_{q}}{[m]_{q}+\delta}\right)_{q}^{m}} \sum_{k=0}^{m}\left[\begin{array}{c}
m \\
k
\end{array}\right]_{q} q^{\frac{k(k-1)}{2}}\left(u-\frac{\gamma}{[m]_{q}+\delta}\right)^{k}\left(\frac{[m]_{q}+\gamma}{[m]_{q}+\delta}-u\right)^{m-k}[k]_{q}^{3} \\
& +\frac{b^{3}}{\left.([m]+b)_{q}\right)^{3}\left(\frac{[m]_{q}}{[m]_{q}+\delta}\right)_{q}^{m}} \sum_{k=0}^{m}\left[\begin{array}{c}
m \\
k
\end{array}\right]_{q} q^{\frac{k(k-1)}{2}}\left(u-\frac{\gamma}{[m]_{q}+\delta}\right)^{k}\left(\frac{[m]_{q}+\gamma}{[m]_{q}+\delta}-u\right)^{m-k} \\
& +\frac{3 a}{\left.([m]+b)_{q}\right)^{3}\left(\frac{[m]_{q}}{[m]_{q}+\delta}\right)_{q}^{m}} \sum_{k=0}^{m}\left[\begin{array}{c}
m \\
k
\end{array}\right]_{q} q^{\frac{k(k-1)}{2}}\left(u-\frac{\gamma}{[m]_{q}+\delta}\right)^{k} \\
& \times\left(\frac{[m]_{q}+\gamma}{[m]_{q}+\delta}-u\right)^{m-k}[k]_{q}^{2} \\
& +\frac{3 a^{2}}{\left.([m]+b)_{q}\right)^{3}\left(\frac{[m]_{q}}{[m]_{q}+\delta}\right)_{q}^{m}} \sum_{k=0}^{m}\left[\begin{array}{c}
m \\
k
\end{array}\right]_{q} q^{\frac{k(k-1)}{2}}\left(u-\frac{\gamma}{[m]_{q}+\delta}\right)^{k} \\
& \times\left(\frac{[m]_{q}+\gamma}{[m]_{q}+\delta}-u\right)^{m-k}[k]_{q} \\
& =D+E+F+G \quad \text { (say). }
\end{aligned}
$$

After calculating the values of $D, E, F$ and $G$, we get

$$
\begin{aligned}
S_{m, q}^{(\gamma, \delta, a, b)}\left(t^{3} ; u\right)= & \left(\frac{[m]_{q}+\delta}{[m]_{q}+b}\right) \\
& \times \frac{q^{6}[m-1]_{q}[m-2]_{q}\left(u-\frac{\gamma}{[m]_{q}+\delta}\right)^{3}}{\left([m]_{q}+b\right)^{2}\left\{\frac{[m]_{q}+\gamma}{[m]_{q}+\delta}-u+q\left(u-\frac{\gamma}{[m]_{q}+\delta}\right)\right\}\left\{\frac{[m]_{q}+\gamma}{[m]_{q}+\delta}-u+q^{2}\left(u-\frac{\gamma}{[m]_{q}+\delta}\right)\right\}} \\
& +(2+3 a+q)\left(\frac{q^{2}[m-1]_{q}}{\left([m]_{q}+b\right)^{2}}\right)\left(\frac{[m]_{q}+\delta}{[m]_{q}+b}\right) \frac{\left(u-\frac{\gamma}{[m]_{q}+\delta}\right)^{2}}{\left\{\frac{[m]_{q}+\gamma}{[m]_{q}+\delta}-u+q\left(u-\frac{\gamma}{[m]_{q}+\delta}\right)\right\}} \\
& +\left(1+3 a^{2}\right)\left(\frac{[m]_{q}+\delta}{\left([m]_{q}+b\right)^{3}}\right)\left(u-\frac{\gamma}{[m]_{q}+\delta}\right) \\
& +\frac{a^{3}}{\left([m]_{q}+b\right)^{3}}+\frac{3 a^{2}\left([m]_{q}+\delta\right)}{\left([m]_{q}+b\right)^{3}} .
\end{aligned}
$$


(5) For $f(t)=t^{4}$, we have

$$
\begin{aligned}
& S_{m, q}^{(\gamma, \delta, a, b)}\left(t^{4} ; u\right) \\
& =\frac{1}{\left(\frac{[m]_{q}}{[m]_{q}+\delta}\right)_{q}^{m}} \sum_{k=0}^{m}\left[\begin{array}{c}
m \\
k
\end{array}\right]_{q} q^{\frac{k(k-1)}{2}}\left(u-\frac{\gamma}{[m]_{q}+\delta}\right)^{k}\left(\frac{[m]_{q}+\gamma}{[m]_{q}+\delta}-u\right)^{m-k}\left(\frac{[k]_{q}+a}{[m]_{q}+b}\right)^{4} \\
& =\frac{1}{\left([m]_{q}+b\right)^{4}\left(\frac{[m]_{q}}{[m]_{q}+\delta}\right)_{q}^{m}} \sum_{k=0}^{m}\left[\begin{array}{c}
m \\
k
\end{array}\right]_{q} q^{\frac{k(k-1)}{2}}\left(u-\frac{\gamma}{[m]_{q}+\delta}\right)^{k} \\
& \times\left(\frac{[m]_{q}+\gamma}{[m]_{q}+\delta}-u\right)^{m-k}\left([k]_{q}+a\right)^{4} \\
& =\frac{1}{\left([m]_{q}+b\right)^{4}\left(\frac{[m]_{q}}{[m]_{q}+\delta}\right)_{q}^{m}} \sum_{k=0}^{m}\left[\begin{array}{c}
m \\
k
\end{array}\right]_{q} q^{\frac{k(k-1)}{2}}\left(u-\frac{\gamma}{[m]_{q}+\delta}\right)^{k}\left(\frac{[m]_{q}+\gamma}{[m]_{q}+\delta}-u\right)^{m-k}[k]_{q}^{4} \\
& +\frac{a^{4}}{\left([m]_{q}+b\right)^{4}\left(\frac{[m]_{q}}{[m]_{q}+\delta}\right)_{q}^{m}} \sum_{k=0}^{m}\left[\begin{array}{c}
m \\
k
\end{array}\right]_{q} q^{\frac{k(k-1)}{2}}\left(u-\frac{\gamma}{[m]_{q}+\delta}\right)^{k}\left(\frac{[m]_{q}+\gamma}{[m]_{q}+\delta}-u\right)^{m-k} \\
& +\frac{6 a^{2}}{\left([m]_{q}+b\right)^{4}\left(\frac{[m]_{q}}{[m]_{q}+\delta}\right)_{q}^{m}} \sum_{k=0}^{m}\left[\begin{array}{c}
m \\
k
\end{array}\right]_{q} q^{\frac{k(k-1)}{2}}\left(u-\frac{\gamma}{[m]_{q}+\delta}\right)^{k}\left(\frac{[m]_{q}+\gamma}{[m]_{q}+\delta}-u\right)^{m-k}[k]_{q}^{2} \\
& +\frac{4 a}{\left([m]_{q}+b\right)^{4}\left(\frac{[m]_{q}}{[m]_{q}+\delta}\right)_{q}^{m}} \sum_{k=0}^{m}\left[\begin{array}{c}
m \\
k
\end{array}\right]_{q} q^{\frac{k(k-1)}{2}}\left(u-\frac{\gamma}{[m]_{q}+\delta}\right)^{k}\left(\frac{[m]_{q}+\gamma}{[m]_{q}+\delta}-u\right)^{m-k}[k]_{q}^{3} \\
& +\frac{4 a^{3}}{\left([m]_{q}+b\right)^{4}\left(\frac{[m]_{q}}{[m]_{q}+\delta}\right)_{q}^{m}} \sum_{k=0}^{m}\left[\begin{array}{c}
m \\
k
\end{array}\right]_{q} q^{\frac{k(k-1)}{2}}\left(u-\frac{\gamma}{[m]_{q}+\delta}\right)^{k}\left(\frac{[m]_{q}+\gamma}{[m]_{q}+\delta}-u\right)^{m-k}[k]_{q} \\
& =H+I+J+K+L \quad \text { (say). }
\end{aligned}
$$

After calculating the values of $H, I, J, K$ and $L$, we obtain

$$
\begin{aligned}
S_{m, q}^{(\gamma, \delta, a, b)}\left(t^{4} ; u\right) & \frac{q^{12}[m-1]_{q}[m-2]_{q}[m-3]_{q}}{\left([m]_{q}+b\right)^{3}}\left(\frac{[m]_{q}+\delta}{[m]_{q}+b}\right) \frac{1}{\left\{\frac{[m]_{q}+\gamma}{[m]_{q}+\delta}-u+q\left(u-\frac{\gamma}{[m]_{q}+\delta}\right)\right\}} \\
& \times \frac{\left(u-\frac{\gamma}{[m]_{q}+\delta}\right)^{4}}{\left\{\frac{[m]_{q}+\gamma}{[m]_{q}+\delta}-u+q^{2}\left(u-\frac{\gamma}{[m]_{q}+\delta}\right)\right\}\left\{\frac{[m]_{q}+\gamma}{[m]_{q}+\delta}-u+q^{3}\left(u-\frac{\gamma}{[m]_{q}+\delta}\right)\right\}} \\
& +\frac{[m-1]_{q}[m-2]_{q}}{\left([m]_{q}+b\right)^{3}}\left(\frac{[m]_{q}+\delta}{[m]_{q}+b}\right) \\
& \times \frac{\left(q^{8}+2 q^{7}+3 q^{5}+4 a q^{2}\right)\left(u-\frac{\gamma}{[m]_{q}+\delta}\right)^{3}}{\left\{\frac{[m]_{q}+\gamma}{[m]_{q}+\delta}-u+q\left(u-\frac{\gamma}{[m]_{q}+\delta}\right)\right\}\left\{\frac{[m]_{q}+\gamma}{[m]_{q}+\delta}-u+q^{2}\left(u-\frac{\gamma}{[m]_{q}+\delta}\right)\right\}} \\
& +\left(a^{4}+3 q^{3}+3 q^{2}+6 a^{2} q^{2}\right) \frac{[m-1]_{q}}{\left([m]_{q}+b\right)^{3}}\left(\frac{[m]_{q}+\delta}{[m]_{q}+b}\right) \frac{\left(u-\frac{\gamma}{[m]_{q}+\delta}\right)^{2}}{\left\{\frac{[m]_{q}+\gamma}{[m]_{q}+\delta}-u+q\left(u-\frac{\gamma}{[m]_{q}+\delta}\right)\right\}} \\
& +\left(1+4 a^{3}\right)\left(\frac{[m]_{q}+\delta}{\left([m]_{q}+b\right)^{4}}\right)\left(u-\frac{\gamma}{[m]_{q}+\delta}\right)+\frac{a^{4}}{\left([m]_{q}+b\right)^{4}}+\frac{6 a^{2}\left([m]_{q}+\delta\right)}{\left([m]_{q}+b\right)^{4}} .
\end{aligned}
$$


Lemma 3.2 By using the linearity of operators $S_{m, q}^{(\gamma, \delta, a, b)}(f ; u)$ and by Lemma 3.1, for all $u \in$ $\left[\frac{\gamma}{[m]_{q}+\delta}, \frac{[m]_{q}+\gamma}{[m]_{q}+\delta}\right]$, we can acquire the central moments as

(1) $S_{m, q}^{(\gamma, \delta, a, b)}((t-u) ; u)=\frac{[m]_{q}+\delta}{[m]_{q}+b}\left(u-\frac{\gamma}{[m]_{q}+\delta}\right)+\frac{a}{[m]_{q}+b}-u$,

(2) $S_{m, q}^{(\gamma, \delta, a, b)}\left((t-u)^{2} ; u\right)=\left(\frac{q^{2}[m-1]_{q}}{[m]_{q}+b}\right)\left(\frac{[m]_{q}+\delta}{[m]_{q}+b}\right) \frac{\left(u-\frac{\gamma}{[m]_{q}+\delta}\right)^{2}}{\left\{\frac{[m]_{q}+\gamma}{[m]_{q}+\delta}-u+q\left(u-\frac{\gamma}{[m]_{q}+\delta}\right)\right\}}+\left(\frac{1}{[m]_{q}+b}-2 u\right)\left(\frac{[m]_{q}+\delta}{[m]_{q}+b}\right)(u-$ $\left.\frac{\gamma}{[m]_{q}+\delta}\right)+\left(\frac{a}{[m]_{q}+b}\right)\left(\frac{1}{[m]_{q}+b}-2 u\right)+u^{2}$

(3) $S_{m, q}^{(\gamma, \delta, a, b)}\left((t-u)^{3} ; u\right)=\left(\frac{[m]_{q}+\delta}{[m]_{q}+b}\right) \frac{q^{6}[m-1]_{q}[m-2]_{q}\left(u-\frac{\gamma}{[m]_{q}+\delta}\right)^{3}}{\left([m]_{q}+b\right)^{2}\left\{\frac{[m]_{q}+\gamma}{[m]_{q}+\delta}-u+q\left(u-\frac{\gamma}{[m]_{q}+\delta}\right)\right\}\left\{\frac{[m]_{q}+\gamma}{[m]_{q}+\delta}-u+q^{2}\left(u-\frac{\gamma}{[m]_{q}+\delta}\right)\right\}}+$

$$
\begin{aligned}
& \left(\frac{2+3 a+q}{\left([m]_{q}+b\right)}-3 u\right)\left(\frac{q^{2}[m-1]_{q}}{\left([m]_{q}+b\right)}\right)\left(\frac{[m]_{q}+\delta}{[m]_{q}+b}\right) \frac{\left(u-\frac{\gamma}{[m]_{q}+\delta}\right)^{2}}{\left\{\frac{m]_{q}+\gamma}{[m]_{q}+\delta}-u+q\left(u-\frac{\gamma}{[m]_{q}+\delta}\right)\right\}}+\left(\frac{1+3 a^{2}}{\left([m]_{q}+b\right)^{2}}-\frac{3 u}{[m]_{q}+b}+3 u^{2}\right) \times \\
& \left(\frac{[m]_{q}+\delta}{[m]_{q}+b}\right)\left(u-\frac{\gamma}{[m]_{q}+\delta}\right)+\frac{a^{3}}{\left([m]_{q}+b\right)^{3}}+3 a^{2}\left(\frac{[m]_{q}+\delta}{\left([m]_{q}+b\right)^{3}}\right)-\frac{3 u^{2} a}{\left([m]_{q}+b\right)^{2}}+3 u^{2}\left(\frac{[m]_{q}+\delta}{[m]_{q}+b}\right)\left(u-\frac{\gamma}{[m]_{q}+\delta}\right)+ \\
& \frac{3 a^{2}}{\left([m]_{q}+b\right)}-u^{3},
\end{aligned}
$$

(4) $S_{m, q}^{(\gamma, \delta, a, b)}\left((t-u)^{4} ; u\right)=\frac{q^{12}[m-1]_{q}[m-2]_{q}[m-3]_{q}}{\left([m]_{q}+b\right)^{3}}\left(\frac{[m]_{q}+\delta}{[m]_{q}+b}\right) \frac{1}{\left\{\frac{m]_{q}+\gamma}{[m]_{q}+\delta}-u+q\left(u-\frac{\gamma}{[m]_{q}+\delta}\right)\right\}} \times$

$$
\begin{aligned}
& \frac{\left(u-\frac{\gamma}{[m]_{q}+\delta}\right)^{4}}{\left\{\frac{m]_{q}+\gamma}{[m]_{q}+\delta}-u+q^{2}\left(u-\frac{\gamma}{[m]_{q}+\delta}\right)\right\}\left(\frac{[m]_{q}+\gamma}{[m]_{q}+\delta}-u+q^{3}\left(u-\frac{\gamma}{[m]_{q}+\delta}\right)\right\}}+\frac{[m-1]_{q}[m-2]_{q}}{\left([m]_{q}+b\right)^{2}}\left(\frac{[m]_{q}+\delta}{[m]_{q}+b}\right) \times \\
& \frac{\left(\frac{q^{8}+3 q^{5}+2 q^{6}+4 a q^{2}}{[m]_{q}}-4 u q^{6}\right)\left(u-\frac{\gamma}{[m]_{q}+\delta}\right)^{3}}{\left\{\frac{[m]_{q}+\gamma}{[m]_{q}+\delta}-u+q\left(u-\frac{\gamma}{[m]_{q}+\delta}\right)\right\}\left\{\frac{[m]_{q}+\gamma}{[m]_{q}+\delta}-u+q^{2}\left(u-\frac{\gamma}{[m]_{q}+\delta}\right)\right\}}+\left(\frac{q^{4}+3 q^{3}+3 q^{2}+6 a^{2} q^{2}}{\left([m]_{q}+b\right)^{2}}-\frac{8 u q^{2}+4 u q^{3}+12 a u q^{2}}{[m]_{q}+b}-\right. \\
& \left.2 u^{2} q^{2}\right) \frac{[m-1]_{q}}{[m]_{q}+b}\left(\frac{[m]_{q}+\delta}{[m]_{q}+b}\right) \frac{\left(u-\frac{\gamma}{[m] q+\delta}\right)^{2}}{\left\{\frac{[m]_{q}+\gamma}{[m]_{q}+\delta}-u+q\left(u-\frac{\gamma}{[m]_{q}+\delta}\right)\right\}}+\left(\frac{1+4 a^{3}}{\left([m]_{q}+b\right)^{3}}-\frac{4 u+12 a^{2} u}{\left([m]_{q}+b\right)^{2}}+\frac{2 u^{2}}{[m]_{q}+b}-4 u^{3}\right) \times \\
& \left(\frac{[m]_{q}+\delta}{[m]_{q}+b}\right)\left(u-\frac{\gamma}{[m]_{q}+\delta}\right)+\left(\frac{3 a^{2}}{[m]_{q}+b}-12 a^{2} u\right)\left(\frac{[m]_{q}+\delta}{\left([m]_{q}+b\right)^{3}}\right)+\frac{a^{4}}{\left([m]_{q}+b\right)^{4}}-\frac{4 a^{3} u}{\left([m]_{q}+b\right)^{3}}- \\
& \frac{4 a u^{2}}{\left([m]_{q}+b\right)^{2}} \frac{4 a u^{3}}{[m]_{q}+b}+u^{4} .
\end{aligned}
$$

We can easily see that $S_{m, q}^{(\gamma, \delta, a, b)}(f ; u)$ are positive linear operators.

\section{Main results}

Firstly, we prove some theorems on the convergence of $S_{m, q}^{(\gamma, \delta, a, b)}(f ; u)$ to $f(u)$.

Theorem 4.1 Let $f \in C[0,1]$ and the sequence $q_{m}$ satisfying $0<q_{m}<1$ such that $q_{m} \rightarrow 1$ as $m \rightarrow \infty$. Then

$$
\lim _{m \rightarrow \infty} \max _{\frac{\gamma}{[m] q_{m}+\delta} \leq u \leq \frac{[m] q_{m}+\gamma}{[m] q_{m}+\delta}}\left|S_{m, q_{m}}^{(\gamma, \delta, a, b)}(f ; u)-f(u)\right|=0 .
$$

Proof From Lemma 3.1, it follows that

$$
\lim _{m \rightarrow \infty} \max _{\frac{\gamma}{[m] q_{m}+\delta} \leq u \leq \frac{[m] q_{m}+\gamma}{[m] q_{m}+\delta}}\left|S_{m, q_{m}}^{(\gamma, \delta, a, b)}\left(t^{i} ; u\right)-u^{i}\right|=0, \quad i=0,1,2 .
$$

Consider the sequence of operators

$$
S_{m}^{*}(f ; u)= \begin{cases}S_{m, q_{m}}^{(\gamma, \delta, a, b)}, & \text { if } \frac{\gamma}{[m]]_{m}+\delta} \leq u \leq \frac{[m]_{q_{m}}+\gamma}{[m]_{q_{m}}+\delta} \\ f(u), & \text { if } u \in\left[0, \frac{\gamma}{[m]_{q_{m}}+\delta}\right] \cup\left[\frac{[m]_{q_{m}}+\gamma}{[m]_{q_{m}}+\delta}, 1\right]\end{cases}
$$

Then obviously

$$
\left\|S_{m}^{*} f-f\right\|=\max _{\frac{\gamma}{[m] q_{m}+\delta} \leq u \leq \leq[m] q_{m}+\gamma}^{[m] q_{m}+\delta}\left|S_{m, q_{m}}^{(\gamma, \delta, a, b)}(f ; u)-f(u)\right|
$$


and using (4.1) we obtain

$$
\lim _{m \rightarrow \infty}\left\|S_{m}^{*} t^{i}-t^{i}\right\|_{C[0,1]}=0, \quad i=0,1,2
$$

Now, by applying the Korovkin theorem [12] (see also [3]) to the sequence of positive linear operators $S_{m}^{*}$, we obtain

$$
\lim _{m \rightarrow \infty}\left\|S_{m}^{*}(f ; u)-f(u)\right\|_{C[0,1]}=0
$$

for every continuous function $f$. Therefore (4.2) gives

$$
\lim _{m \rightarrow \infty} \max _{\frac{\gamma}{[m] q_{m}+\delta} \leq u \leq \frac{[m] q_{m}+\gamma}{[m] q_{m}+\delta}}\left|S_{m, q_{n}}^{(\gamma, \delta, a, b)}(f ; u)-f(u)\right|=0,
$$

and the proof is completed.

Theorem 4.2 Iff be a continuous function on $[0,1]$ and taking $0<q<1$, then

$$
\left\|S_{m, q}^{(\gamma, \delta, a, b)} f-f\right\| \leq 2 \omega_{f}\left(\sigma_{m}\right)
$$

where

$$
\sigma_{m}=\sqrt{\left.S_{m, q}^{(\gamma, \delta, a, b)}\left((t-u)^{2} ; u\right)\right)} .
$$

Proof For any $u, y \in[a, b]$, it is well known that

$$
|f(y)-f(u)| \leq \omega_{f}(\sigma)\left(1+\frac{(y-u)^{2}}{\sigma^{2}}\right)
$$

Therefore, we get

$$
\begin{aligned}
\left|S_{m, q}^{(\gamma, \delta, a, b)}(f ; u)-f(u)\right| & \leq S_{m, q}^{(\gamma, \delta, a, b)}(|f(t)-f(u)| ; u) \\
& \leq \omega_{f}(\sigma)\left(1+\frac{1}{\sigma^{2}} S_{m, q}^{(\gamma, \delta, a, b)}\left((t-u)^{2} ; u\right)\right) .
\end{aligned}
$$

Choosing $\sigma=\sigma_{m}=\sqrt{\left.S_{m, q}^{(\gamma, \delta, a, b)}\left((t-u)^{2} ; u\right)\right)}$, we have

$$
\left\|S_{m, q}^{(\gamma, \delta, a, b)} f-f\right\| \leq 2 \omega_{f}\left(\sigma_{m}\right) .
$$

Thus, we obtain the desired result.

Theorem 4.3 (Voronovskaja type theorem) Let $f^{\prime \prime} \in C[0,1]$ and $\left(q_{m}\right)_{m \in \mathbb{N}} \subseteq(0,1)$ be a sequence such that $q_{m} \rightarrow 1$ as $m \rightarrow \infty$ and $q_{m}^{m} \rightarrow 0$ as $m \rightarrow \infty$. Then

$$
\lim _{m \rightarrow \infty}[m]_{q_{m}}\left(S_{m, q_{m}}^{(\gamma, \delta, a, b)}(f ; u)-f(u)\right)=(\delta u-\gamma) f^{\prime}(u)+\frac{1}{2}\left(u-\delta u^{2}\right) f^{\prime \prime}(u)
$$

uniformly on $\left[\frac{\gamma}{[m]_{q_{m}}+\delta}, \frac{[m]_{q_{m}}+\gamma}{[m]_{q_{m}}+\delta}\right]$. 
Proof By the Taylor formula we may write

$$
f(t)=f(u)+f^{\prime}(u)(t-u)+\frac{1}{2} f^{\prime \prime}(u)(t-u)^{2}+r(t, u)(t-u)^{2},
$$

where $r(t, u)$ is the remainder term and $\lim _{t \rightarrow u} r(t, u)=0$. Applying $S_{m, q_{m}}^{(\gamma, \delta, a)}(f ; u)$ to (4.3), we obtain

$$
\begin{aligned}
{[m]_{q_{m}}\left(S_{m, q_{m}}^{(\gamma, \delta, a, b)}(f ; u)-f(u)\right)=[} & {[m]_{q_{m}} S_{m, q_{m}}^{(\gamma, \delta, a, b)}((t-u) ; u) f^{\prime}(u) } \\
& +[m]_{q_{m}} S_{m, q_{m}}^{(\gamma, \delta, a, b)}\left((t-u)^{2} ; u\right) \frac{f^{\prime \prime}(u)}{2} \\
& +[m]_{q_{m}} S_{m, q_{m}}^{(\gamma, \delta, a, b)}\left(r(t, u)(t-u)^{2} ; u\right) .
\end{aligned}
$$

By the Cauchy-Schwartz inequality, we have

$$
S_{m, q_{m}}^{(\gamma, \delta, a, b)}\left(r(t, u)(t-u)^{2} ; u\right) \leq \sqrt{S_{m, q_{m}}^{(\gamma, \delta, a, b)}\left(r^{2}(t, u) ; u\right)} \times \sqrt{S_{m, q_{m}}^{(\gamma, \delta, a, b)}\left((t-u)^{4} ; u\right)} .
$$

Observe that $r^{2}(u, u)=0$ and $r^{2}(\cdot, u) \in C[0,1]$, then it follows from Theorem 4.2 that

$$
\lim _{m \rightarrow \infty} S_{m, q_{m}}^{(\gamma, \delta, b)}\left(r^{2}(t, u) ; u\right)=r^{2}(u, u)=0
$$

uniformly with respect to $u \in\left[\frac{\gamma}{[m]_{q m}+\delta}, \frac{[m]_{q_{m}}+\gamma}{[m]_{q_{m}}+\delta}\right]$. From (4.4) and (4.5), we get

$$
\lim _{m \rightarrow \infty}[m]_{q_{m}} S_{m, q_{m}}^{(\gamma, \delta, a, b)}\left(r(t, u)(t-u)^{2} ; q ; u\right)=0 .
$$

Now we compute the following:

$$
\lim _{m \rightarrow \infty}[m]_{q_{m}} S_{m, q_{m}}^{(\gamma, \delta, a, b)}((t-u) ; u)=\delta u-\gamma
$$

uniformly in $\left[\frac{\gamma}{[m]_{q_{m}}+\delta}, \frac{[m]_{q_{m}}+\gamma}{[m]_{q_{m}}+\delta}\right]$.

Finally using the above two equalities, we have

$$
\begin{aligned}
\lim _{m \rightarrow \infty}[m]_{q_{m}}\left(S_{m, q_{m}}^{(\gamma, \delta, a, b)}(f ; u)-f(u)\right)= & \lim _{m \rightarrow \infty}[m]_{q_{m}} S_{m, q_{m}}^{(\gamma, \delta, a, b)}((t-u) ; u) f^{\prime}(u) \\
& +\lim _{m \rightarrow \infty}[m]_{q_{m}} S_{m, q_{m}}^{(\gamma, \delta, a, b)}\left((t-u)^{2} ; u\right) \frac{f^{\prime \prime}(u)}{2} \\
& +\lim _{m \rightarrow \infty}[m]_{q_{m}} S_{m, q_{m}}^{(\gamma, \delta, a, b)}\left(r(t, u)(t-u)^{2} ; u\right) \\
= & (\delta u-\gamma) f^{\prime}(u)+\frac{1}{2}\left(u-\delta u^{2}\right) f^{\prime \prime}(u) .
\end{aligned}
$$

This completes the proof of the theorem.

\section{Local approximation}

If $\sigma>0$ and $W^{2}=\left\{s \in C[0,1] ; s^{\prime}, s^{\prime \prime} \in C[0,1]\right\}$, then the $K$-functional is defined as

$$
K_{2}(f, \sigma)=\inf \left\{\|f-s\|+\sigma\left\|s^{\prime \prime}\right\|\right\} .
$$


By [7], p. 177, Theorem 2.4, there exists an absolute constant $C>0$ such that

$$
K_{2}(f, \sigma) \leq C \omega_{2}(f, \sqrt{\sigma})
$$

where the second order modulus of smoothness for $f \in C[0,1]$ is defined as

$$
\omega_{2}(f, \sqrt{\sigma})=\sup _{0<h \leq \sqrt{\sigma} u} \sup _{u+2 h \in[0,1]}|f(u+2 h)-2 f(u+h)+f(u)| .
$$

The usual modulus of continuity for $f \in C[0,1]$ is defined as

$$
\omega(f, \sigma)=\sup _{0<h \leq \sqrt{\sigma} u} \sup _{u+h \in[0,1]}|f(u+h)-f(u)|
$$

Our next main result is the following local approximation theorem.

Theorem 5.1 Let $f$ be a continuous function on $[0,1]$ with $0<q<1$. Then, for every $u \in$ $\left[\frac{\gamma}{[m]_{q}+\delta}, \frac{[m]_{q}+\gamma}{[m]_{q}+\delta}\right]$, we have

$$
\left|S_{m, q}^{(\gamma, \delta, a, b)}(f ; u)-f(u)\right| \leq C \omega_{2}\left(f, \sigma_{m}(u)\right)+\omega\left(f,\left|\frac{\delta u-\gamma}{[m]_{q}+b}\right|\right),
$$

where

$$
\begin{aligned}
\sigma_{m}^{2}(u)= & \left(\frac{q^{2}[m-1]_{q}}{[m]_{q}+b}\right)\left(\frac{[m]_{q}+\delta}{[m]_{q}+b}\right) \frac{\left(u-\frac{\gamma}{[m]_{q}+\delta}\right)^{2}}{\left\{\frac{[m]_{q}+\gamma}{[m]_{q}+\delta}-u+q\left(u-\frac{\gamma}{[m]_{q}+\delta}\right)\right\}} \\
& +\left(\frac{1}{[m]_{q}+b}-2 u\right)\left(\frac{[m]_{q}+\delta}{[m]_{q}+b}\right)\left(u-\frac{\gamma}{[m]_{q}+\delta}\right) \\
& +\left(\frac{a}{[m]_{q}+b}\right)\left(\frac{1}{[m]_{q}+b}-2 u\right)+u^{2} \\
& +\left(\frac{[m]_{q}+\delta}{[m]_{q}}\left(u-\frac{\gamma}{[m]_{q}+\delta}\right)-u\right)^{2} .
\end{aligned}
$$

Proof We define

$$
\bar{S}_{m, q}^{(\gamma, \delta, a, b)}(f ; u)=S_{m, q}^{(\gamma, \delta, a, b)}(f ; u)+f(u)-f\left(\frac{[m]_{q}+\delta}{[m]_{q}+b}\left(u-\frac{\gamma}{[m]_{q}+\delta}\right)\right) .
$$

From Lemma 3.1, we find

$$
\bar{S}_{m, q}^{(\gamma, \delta, a, b)}(1 ; u)=S_{m, q}^{(\gamma, \delta, a, b)}(1 ; u)=1
$$

and

$$
\bar{S}_{m, q}^{(\gamma, \delta, a, b)}(t ; u)=S_{m, q}^{(\gamma, \delta, a, b)}(t ; u)+u-\left(\frac{[m]_{q}+\delta}{[m]_{q}+b}\right)\left(u-\frac{\gamma}{[m]_{q}+\delta}\right)=u .
$$

Let $s \in W^{2}$. By using Taylor's formula we have

$$
s(t)=s(u)+(t-u) s^{\prime}(u)(t-v) s^{\prime \prime}(v) d v,
$$


Mursaleen et al. Journal of Inequalities and Applications

(2020) $2020: 28$

Page 12 of 14

we get

$$
\begin{aligned}
& \bar{S}_{m, q}^{(\gamma, \delta, a, b)}(s ; u) \\
& =s(u)+s^{\prime}(u) \bar{S}_{m, q}^{(\gamma, \delta, a, b)}((t-u) ; u)+\bar{S}_{m, q}^{(\gamma, \delta, a, b)}\left(\int_{u}^{t}(t-v) s^{\prime \prime}(v) d v ; u\right) \\
& =s(u)+S_{m, q}^{(\gamma, \delta, a, b)}\left(\int_{u}^{t}(t-v) s^{\prime \prime}(v) d v ; u\right) \\
& \quad-\int_{u}^{\frac{[m] q+\delta}{[m]_{q}+b}\left(u-\frac{\gamma}{[m]_{q}+\delta}\right)}\left[\frac{[m]_{q}+\delta}{[m]_{q}+b}\left(u-\frac{\gamma}{[m]_{q}+\delta}\right)-v\right] s^{\prime \prime}(v) d v,
\end{aligned}
$$

which implies that

$$
\begin{aligned}
& \left|\bar{S}_{m, q}^{(\gamma, \delta, a, b)}(s ; u)-s(u)\right| \\
& \leq S_{m, q}^{(\gamma, \delta, a, b)}\left(\left|\int_{u}^{t}(t-v) s^{\prime \prime}(v) d v\right| ; u\right) \\
& \quad+\left|\int_{u}^{\frac{[m]]^{+\delta}}{[m] q+b}\left(u-\frac{\gamma}{[m] q+\delta}\right)}\right|\left[\frac{[m]_{q}+\delta}{[m]_{q}+b}\left(u-\frac{\gamma}{[m]_{q}+\delta}\right)-v\right]\left|s^{\prime \prime}(v)\right| d v \mid \\
& \leq S_{m, q}^{(\gamma, \delta, a, b)}\left((t-u)^{2} ; u\right)\left\|s^{\prime \prime}\right\|+\left[\frac{[m]_{q}+\delta}{[m]_{q}+b}\left(u-\frac{\gamma}{[m]_{q}+\delta}\right)-u\right]^{2}\left\|s^{\prime \prime}\right\| .
\end{aligned}
$$

From Lemma 3.2(1), we have

$$
\begin{aligned}
\left|\bar{S}_{m, q}^{(\gamma, \delta, a, b)}(s ; u)-s(u)\right| & \left(\left(\frac{q^{2}[m-1]_{q}}{[m]_{q}+b}\right)\left(\frac{[m]_{q}+\delta}{[m]_{q}+b}\right) \frac{\left(u-\frac{\gamma}{[m]_{q}+\delta}\right)^{2}}{\left\{\frac{[m]_{q}+\gamma}{[m]_{q}+\delta}-u+q\left(u-\frac{\gamma}{[m]_{q}+\delta}\right)\right\}}\right. \\
& +\left(\frac{1}{[m]_{q}+b}-2 u\right)\left(\frac{[m]_{q}+\delta}{[m]_{q}+b}\right)\left(u-\frac{\gamma}{[m]_{q}+\delta}\right) \\
& \left.+\left(\frac{a}{[m]_{q}+b}\right)\left(\frac{1}{[m]_{q}+b}-2 u\right)+u^{2}\right)\left\|s^{\prime \prime}\right\| \\
& +\left(\frac{[m]_{q}+\delta}{[m]_{q}+b}\left(u-\frac{\gamma}{[m]_{q}+\delta}\right)-u\right)^{2}\left\|s^{\prime \prime}\right\| \\
\leq & \left\{\left(\frac{q^{2}[m-1]_{q}}{[m]_{q}+b}\right)\left(\frac{[m]_{q}+\delta}{[m]_{q}+b}\right) \frac{\left(u-\frac{\gamma}{[m]_{q}+\delta}\right)^{2}}{\left\{\frac{[m]_{q}+\gamma}{[m]_{q}+\delta}-u+q\left(u-\frac{\gamma}{[m]_{q}+\delta}\right)\right\}}\right. \\
& +\left(\frac{1}{[m]_{q}+b}-2 u\right)\left(\frac{[m]_{q}+\delta}{[m]_{q}+b}\right)\left(u-\frac{\gamma}{[m]_{q}+\delta}\right) \\
& \left.+\left(\frac{a}{[m]_{q}+b}\right)\left(\frac{1}{[m]_{q}+b}-2 u\right)+u^{2}\right) \\
& \left.+\left(\frac{[m]_{q}+\delta}{[m]_{q}+b}\left(u-\frac{\gamma}{[m]_{q}+\delta}\right)-u\right)^{2}\right\}\left\|s^{\prime \prime}\right\| \\
= & \sigma_{m}^{2}(u)\left\|s^{\prime \prime}\right\| \cdot
\end{aligned}
$$


On the other hand, we have

$$
\left|\bar{S}_{m, q}^{(\gamma, \delta, a, b)}(f ; u)\right| \leq 3\|f\|
$$

Now, for $f \in C[0,1]$ and $s \in W^{2}$, by using (5.7) and (5.8), we get

$$
\begin{aligned}
& \left|S_{m, q}^{(\gamma, \delta, a, b)}(f ; u)-f(u)\right| \\
& \leq\left|\bar{S}_{m, q}^{(\gamma, \delta, a, b)}(f ; u)-f(u)+f\left(\frac{[m]_{q}+\delta}{[m]_{q}+b}\left(u-\frac{\gamma}{[m]_{q}+\delta}\right)\right)-f(u)\right| \\
& \leq\left|\bar{S}_{m, q}^{(\gamma, \delta, a, b)}(f-s ; u)\right|+\left|\bar{S}_{m, q}^{(\gamma, \delta, a, b)}(s ; u)-s(u)\right| \\
& \quad+|s(u)-f(u)|+\left|f\left(\frac{\left([m]_{q}+\delta\right) u-\gamma}{[m]_{q}+b}\right)-f(u)\right| \\
& \leq 4\|f-s\|+\sigma_{m}^{2}(u)\left\|s^{\prime \prime}\right\|+\left|f\left(\frac{\left([m]_{q}+\delta\right) u-\gamma}{[m]_{q}+b}\right)-f(u)\right| .
\end{aligned}
$$

Taking the infimum on the right hand side over all $s \in W^{2}$, we obtain

$$
\begin{aligned}
\left|S_{m, q}^{(\gamma, \delta, a, b)}(f ; u)-f(u)\right| & \leq 4 K_{2}\left(f, \sigma_{m}^{2}(u)\right)+\omega\left(f,\left|\frac{\left([m]_{q}+\delta\right) u-\gamma}{[m]_{q}+b}-u\right|\right) \\
& =4 K_{2}\left(f, \sigma_{m}^{2}(u)\right)+\omega\left(f,\left|\frac{\delta u-\gamma}{[m]_{q}+b}\right|\right) .
\end{aligned}
$$

Now using (5.3), we have

$$
\left|S_{m, q}^{(\gamma, \delta, a, b)}(f ; u)-f(u)\right| \leq C \omega_{2}\left(f, \sigma_{m}(u)\right)+\omega\left(f,\left|\frac{\delta u-\gamma}{[m]_{q}+b}\right|\right) .
$$

This completes the proof.

\section{Conclusion}

It can be concluded that the parameters $\gamma, \delta, q, a$ and $b$ will provide more modeling flexibility for approximation of functions and bases of these operators can be used to draw curves and surfaces in CAGD. Also the approximation results derived for shifted intervals will be very helpful when it comes to implementation using computers for simulation purposes.

\section{Acknowledgements}

The authors are extremely thankful to the referees for their valuable comments and suggestion. The second author is also grateful to Council of Scientific and Industrial Research (CSIR), India, for providing the Senior Research Fellowship (09/1172(0001)/2017-EMR-I).

\section{Funding}

NA.

\section{Availability of data and materials}

NA.

Competing interests

The authors declare that they have no competing interests. 
Authors' contributions

The authors contributed equally and significantly in writing this paper. All authors read and approved the final manuscript.

\section{Author details}

1 Department of Medical Research, China Medical University Hospital, China Medical University (Taiwan), Taichung, Taiwan. ${ }^{2}$ Department of Computer Science and Information Engineering, Asia University, Taichung, Taiwan. ${ }^{3}$ Department of Mathematics, Aligarh Muslim University, Aligarh, India. ${ }^{4}$ Department of Mathematical Sciences, Baba Ghulam Shah Badshah University, Rajouri, India.

\section{Publisher's Note}

Springer Nature remains neutral with regard to jurisdictional claims in published maps and institutional affiliations.

\section{Received: 25 November 2019 Accepted: 29 January 2020 Published online: 04 February 2020}

\section{References}

1. Acar, T.: Quantitative $q$-Voronovskaya, $q$-Grüss-Voronovskaya-type results for $q$-Szasz operators. Georgian Math. J. 23(4), 459-468 (2016)

2. Acar, T., Aral, A., Gupta, V.: On approximation properties of a new type Bernstein-Durrmeyer operators. Math. Slovaca 65(5), 1107-1122 (2015)

3. Altomare, F., Campiti, M.: Korovkin-Type Approximation Theory and Its Applications. De Gruyter Studies in Mathematics, vol. 17. de Gruyter, Berlin (1994)

4. Aral, A., Acar, T.: Weighted approximation by new Bernstein-Chlodowsky-Gadjiev operators. Filomat 27(2), 373-382 (2013)

5. Bernstein, S.N.: Démonstation du théorème de Weierstrass fondée sur le calcul de probabilités. Commun. Soc. Math. Kharkow 13, 1-2 (1912-1913)

6. Derriennic, M.M.: Modified Bernstein polynomials and Jacobi polynomials in q-calculus. Rend. Circ. Mat. Palermo 76 269-290 (2005)

7. DeVore, R.A., Lorentz, G.G.: Constructive Approximation. Springer, Berlin (1993)

8. Gadjiev, A.D., Ghorbanalizadeh, A.M.: Approximation properties of a new type Bernstein-Stancu polynomials of one and two variables. Appl. Math. Comput. 216, 890-901 (2010)

9. Kac, V., Cheung, P.: Quantum Calculus. Springer, New York (2002)

10. Kajla, A., Acar, T.: A new modification of Durrmeyer type mixed hybrid operators. Carpath. J. Math. 34(1), 47-56 (2018)

11. Khan, K., Lobiyal, D.K., Kilicman, A.: Bézier curves and surfaces based on modified Bernstein polynomials. Azerb. J. Math. 9(1) (2019)

12. Korovkin, P.P.: On convergence of linear operators in the space of continuous functions (Russian). Dokl. Akad. Nauk SSSR 90, 961-964 (1953)

13. Lorentz, G.G.: Bernstein Polynomials. University of Toronto Press, Toronto (1953)

14. Lupaş, A.: A q-analogue of the Bernstein operator. In: Seminar on Numerical and Statistical Calculus. University of Cluj-Napoca, vol. 9, pp. 85-92 (1987)

15. Mishra, V.N., Patel, P.: On generalized integral Bernstein operators based on q-integers. Appl. Math. Comput. 242, 931-944 (2014)

16. Mohiuddine, S.A., Acar, T., Alghamdi, M.A.: Genuine modified Bernstein-Durrmeyer operators. J. Inequal. Appl. 2018 104 (2018)

17. Mohiuddine, S.A., Acar, T., Alotaib, A.: Construction of a new family of Bernstein-Kantorovich operators. Math. Methods Appl. Sci. 40(18), 7749-7759 (2017)

18. Montano, M.C., Leonessa, V.: A sequence of Kantorovich-type operators on mobile intervals. Constr. Math. Anal. 2(3), 130-143 (2019)

19. Mursaleen, M., Ahasan, M.: The Dunkl generalization of Stancu type q-Szász-Mirakjan-Kantrovich operators and some approximation results. Carpath. J. Math. 34(3), 363-370 (2018)

20. Mursaleen, M., Ansari, K.J., Khan, A.: Approximation properties and error estimation of $q$-Bernstein shifted operators. Numer. Algorithms (2019). https://doi.org/10.1007/s11075-019-00752-4

21. Mursaleen, M., Khan, T.: On approximation by Stancu type Jakimovski-Leviatan-Durrmeyer operators. Azerb. J. Math. 7(1), 16-26 (2017)

22. Mursaleen, M., Rahman, S.: Dunkl generalization of $q$-Szász-Mirakjan operators which preserve $x^{2}$. Filomat 32(3) (2018)

23. Phillips, G.M.: Bernstein polynomials based on the q-integers. Ann. Numer. Math. 4, 511-518 (1997)

24. Qasim, M., Mursaleen, M., Khan, A., Abbas, A.: Approximation by generalized Lupaş operators based on q-integers. Mathematics 8(1), Article 68 (2020)

25. Rao, N., Wafi, A., Acu, A.M.: q-Szász-Durrmeyer type operators based on Dunkl analogue. Complex Anal. Oper. Theory https://doi.org/10.1007/s11785-018-0816-3

26. Stancu, D.D.: Approximation of functions by a new class of linear polynomial operators. Rev. Roum. Math. Pures Appl. 13, 1173-1194 (1968) 